\title{
Locus Coeruleus Neurons in Monkey Are Selectively Activated by Attended Cues in a Vigilance Task
}

\author{
Gary Aston-Jones, ${ }^{1}$ Janusz Rajkowski, ${ }^{1}$ Piotr Kubiak, ${ }^{1}$ and Tatiana Alexinsky ${ }^{2}$ \\ 'Division of Behavioral Neurobiology, Department of Mental Health Sciences, Hahnemann University, Philadelphia, \\ Pennsylvania 19102 and Institute des Neurosciences, CNRS, Universite P \& M Curie, Paris 75005, France
}

Impulse activity was recorded extracellularly from noradrenergic neurons in the nucleus locus coeruleus (LC; 47 single-cell and 126 multicell recordings) of four cynomolgus monkeys performing an oddball visual discrimination task. For juice reward, the subjects were required to release a lever rapidly in response to an infrequent (10-20\% of trials) target cue $(C S+)$ that was randomly intermixed with nontarget (CS - ) stimuli presented on a video display. All LC neurons examined were phasically and selectively activated by target cues in this task. Other task events elicited no consistent response from these neurons (juice reward, lever release, fix-spot stimuli, nontarget stimuli). In one animal, nontarget cues phasically inhibited LC neurons. Phasic LC excitatory responses to target cues in this task occurred at a relatively short latency (mean $=90.7 \mathrm{msec}$ ), approximately $200 \mathrm{msec}$ prior to the behavioral response (lever release). In addition, LC response magnitudes varied with behavioral performance, being substantially attenuated during epochs of poor performance (high false alarm rate). There was a positive correlation $(r=0.30, p<0.0001)$ between the latency of LC responses and the latency of behavioral responses to same target cues, consistent with the possibility that $L C$ responses may have a role in selective attention by facilitating responses to the CS + stimulus. Analyses of behavioral response latencies to pairs of stimuli indicated that LC responses may facilitate behavioral responses to subsequent sensory cues, consistent with a role of this system in sustained attention/vigilance. Moreover, responses became reduced in magnitude over time during prolonged task performance (>90 min), in parallel with a behavioral performance decrement.

These results show that LC neurons are activated selectively by attended stimuli that demand a rapid response in this task, and that such LC responses may contribute to conditioned behavioral responses.

\footnotetext{
Received Oct. 20, 1993; revised Jan. 12, 1994; accepted Jan. 27, 1994.

We thank Dr. Y. Zhu for histological processing; Drs. Jon Druhan and Joe LeDoux for comments on the manuscript; Drs. Bob Desimone, David L. Robinson, Michael Goldberg, Randall Nelson, and Michael Posner for helpful advice: and $\mathrm{L}$. Work and S. Aston-Jones for graphic and typographical assistance. This research was sponsored by the Air Force Office of Scientific Research, Air Force Systems Command, USAF, under Grants AFOSR-90-0147 and F49620-93-10099. The U.S. Government is authorized to reproduce and distribute reprints for governmental purposes notwithstanding any copyright notation thereon.

Correspondence should be addressed to Gary Aston-Jones, Ph.D., Division of Behavioral Neurobiology, Department of Mental Health Sciences, Hahnemann University, MS 403, Broad and Vine, Philadelphia, PA 19102.

Copyright (C) 1994 Society for Neuroscience $0270-6474 / 94 / 144467-14 \$ 05.00 / 0$
}

[Keywords: locus coeruleus, noradrenergic, vigilance, unit recording, conditioned stimulus, norepinephrine, attention, primate, conditioning, sensory response

The noradrenergic brain nucleus locus coeruleus (LC), located in the rostral pontine tegmentum, has been the object of intense study since it was found to be the largest nucleus of norepinephrine (NE) neurons in the brain (Dahlstrom and Fuxe, 1964; Moore and Bloom, 1979). This set of neurons has a uniquely diverse set of efferent projections that innervate all levels of the central neuraxis, and provides the sole NE innervation of the cerebral, limbic, and cerebellar cortices (Ungerstedt, 1971; Freedman et al., 1975; Garver and Sladek, 1976). Studies based upon lesion and pharmacologic manipulations have led to several hypotheses for functions of this system, including control of the sleep-waking cycle (Jouvet, 1969), regulation of learning and memory (Crow and Wendlandt, 1976; Everitt et al., 1983; Mohammed et al., 1986; Harris and Fitzgerald, 1991), control of certain autonomic functions (Ward and Gunn, 1976; Miyawaki et al., 1991, 1993), and contribution to affective state (Siever and Davis, 1985; Valentino and (urtis, 1991), including anxiety (Redmond, 1979). However, such studies have generated little consensus concerning LC function (Aston-Jones et al., 1984).

Our investigations of this system have centered upon the anatomical and physiological attributes of LC, neurons (AstonJones et al., 1991a,b). A major goal of these studies has been to characterize the impulse activity of LC neurons in behaviorally active animals. In rats, tonic LC impulse activity was found to vary in association with changes in behavioral state, such that highest tonic discharge occurred with alertness and phasic interaction with external stimuli, while lower tonic activity accompanied behaviors characterized by lower vigilance (e.g., sleep, grooming, drinking) (Aston-Jones and Bloom, 1981). In addition, LC neurons in alert rodents were markedly responsive to sensory stimuli of a variety of modalities (AstonJones and Bloom, 1981). Unconditioned auditory, visual, or tactile stimuli phasically activated LC neurons. Furthermore, the magnitude of activation varied with behavioral state, so that largest responses were elicited by stimuli that disrupted lowvigilance behavior and evoked a behavioral orienting response, while similar stimuli that elicited no behavioral reaction also evoked a much smaller amplitude of phasic LC activation. Similar results have been observed in cat (Rasmussen et al., 1986; Reiner, 1986) and monkey LC neurons (Foote et al., 1980; Grant et al., 1988). In light of the broad efferent projections of the LC and the modulatory effects of NE on LC target neurons, these findings led to the hypothesis that the NE-LC system is primarily 
involved in regulating attention to the external environment and readiness to respond to unexpected stimuli and events (Aston-Jones and Bloom, $1981 \mathrm{a}, \mathrm{b}$; Aston-Jones et al., 1984, 1991; Aston-Jones, 1985).

More recent studies have extended these findings by showing that LC neurons are activated by stimuli that are not in themselves intense or conspicuous, but that are salient to the animal by virtue of conditioning. One such study (Rasmussen and Jacobs, 1986) investigated LC activity in cats during a conditioned emotional response paradigm in which a lone (conditioned stimulus; $C S$ ) was associated with an aversive air puff (unconditioned stimulus). After such pairing the CS alone potently activated $\mathrm{LC}$ neurons. A recent study in rat found that responses of $\mathrm{LC}$ neurons were enhanced for sensory cues that were classically conditioned by association with food or footshock (Sara and Segal, 1991). These findings for conditioned stimulus effects on $\mathrm{LC}$ activity are consistent with the view that the NE-LC system alters attention to sensory events. It is noteworthy, however, that there has been no previous study of LC neuronal activity in animals performing an operant behavioral task in which attention is measured. This is important, as the attentional demands in an operant task involving instrumental responding are quite different from those in passive classical conditioning or conditioned emotional response tasks. In particular, as no behavioral response is required in such passive tasks they do not test the involvement of the $\mathrm{LC}$ in regulating readiness to respond to sensory events. Thus, recordings from LC neurons during instrumental conditioning tasks may provide additional insight into the role of the $\mathrm{LC}$ in attention.

One framework within which to examine attentiveness to environmental stimuli is provided by so-called "oddball" (or continuous performance) tasks developed by experimental psychologists in studies of sustained attention in humans. In these studies, vigilance (synonymous with sustained attention) is defined as a measure of the ability to detect and respond appropriately to infrequent target $(\mathrm{CS}+)$ stimuli embedded among frequent nontarget (CS-) cues (for review, see Davies and Parasuraman, 1982; Warm, 1984). Such studies were initially developed to study watchkeeping behavior of railway workers and radar operators (N. H. Mackworth, 1948, 1957; J. F. Mackworth, 1970).

Here, we used an analogous approach to investigate the role of the LC system in vigilance and adaptive behavioral responsiveness. We recorded activity of single LC neurons while monkeys performed a visual oddball discrimination task, similar to those used in humans to test a subject's ability to detect and respond to unpredictable, infrequent target cues embedded among more common stimuli. Results indicate that noradrenergic LC neurons in the primate are selectively and phasically activated by target $(\mathrm{CS}+)$ cues in this task, and that such activation varies with the level of task performance.

\section{Materials and Methods}

Behavioral training. Subjects were four adult cynomolgus monkeys ( $\mathrm{Ma}$ caca fascicularis). Each animal was habituated to a customized restraining device consisting of a cubicle in which the monkey's body was placed, and a neck yoke through which the animal's head extended. This device allowed free trunk and limb movement, and was adjusted for maximum comfort for each individual animal. Monkeys voluntarily entered this device for daily sessions soon after initial habituation.

Training and experimental recording sessions took place in a soundinsulated wooden enclosure (two animals) or an acoustically insulated, electrically shielded metal chamber (IAC, Inc.). Both enclosures con- tained a video camera for remotely monitoring behavior. The wooden enclosure contained a device for delivering differently colored cue lights (yellow, red, or green; Tri-Colored "Q" Lamp, BRS/LVE) located approximately $40 \mathrm{~cm}$ in front of the animal. The metal chamber contained a color video monitor situated about $25 \mathrm{~cm}$ in front of the animal for stimulus presentation (monitor screen $=58^{\circ} \times 46^{\circ}$ of visual angle). Monkeys were trained in successive stages to depress a lever to initiate stimulus presentation, and then to release it selectively in response to the stimulus that served as the target cue $(\mathrm{CS}+)$ to receive a drop of juice reward (Tang). Responses to the other stimulus (nontarget cue; CS-) were not reinforced with Tang, but instead generated a $3 \mathrm{sec}$ timeout period. Conditioned cues were either the colored lights or horizontal or vertical line segments on the video monitor $\left(0.7^{\circ} \times 2^{\circ}\right.$ of visual angle, white on a dark background). Training continued until animals performed at a level of at least $85 \%$ correct (no, or nearly no, misses and less than $15 \%$ incorrect releases to nontarget stimuli) with a target: nontarget ratio of $20 \%$. Animals were then subjected to implantation surgery.

Implantation surgery. Surgeries were performed using sterile techniques after $12 \mathrm{hr}$ of food deprivation. Anesthesia was induced with 15 $\mathrm{mg} / \mathrm{kg}$ (i.m.) ketamine and an inflatable endotracheal tube was secured. Anesthesia was maintained with a $0.5-1.5 \%$ halothane-air mixture administered through this endotracheal tube with spontaneous respiration. The EKG, heart rate, respiration, and rectal temperature were continuously monitored.

Two types of recording implants were used in these experiments. In the early experiments (colored cue lights), animals were implanted bilaterally with bundles of microwire electrodes directed toward the LC. These wire bundles were mounted in separate microdrives (for each hemisphere) that were fixed to the skull with stainless steel screws reinforced by dental acrylic. These microdrives allowed vertical movement of the wire bundles over a $10 \mathrm{~mm}$ range within a single penetration. Each microdrive contained four bundles of six $25-\mu$ m-diameter stainless steel wires, with the bundles arranged in a $1 \mathrm{~mm}$ square pattern.

Animals tested with stimuli on the video monitor had their heads fixed in place. This was accomplished by cementing a solid aluminum post securely to the skull using specially designed bone anchors and stainless steel bone screws. This post was clamped inside a sleeve attached to the primate chair, providing easy fixation of the animal's head during final training and all recording sessions. The $\mathrm{LC}$ recording implant for these animals consisted of a $1-\mathrm{mm}$-diameter guide cannula that was stereotaxically positioned through a $2 \mathrm{~mm}$ hole in the skull and aimed at the LC of one hemisphere. The lower end of this guide cannula was positioned approximately $5 \mathrm{~mm}$ above the LC, and the top end was attached firmly (but not permanently) within a cylindrical pedestal, which was fixed to the skull, bone anchors, and screws with dental cement. Thus, for $L C$ recordings a single cannula passed through the occipital cortex, the colliculi, and the rostral tip of the cerebellum. Such implants have caused no performance deficit on vigilance tasks, or any other behavioral anomaly in our studies. The recording implant allowed for small changes in the angle of the guide cannula without removal, so that multiple recording penetrations with minimal damage were possible with each implant.

For the electroencephalogram (EEG) recordings, screw clcctrodes $(0-$ 80 gauge, $6 \mathrm{~mm}$ length) with attached wire leads were threaded through the skull at locations analogous to those in the International 10-20 Electrode Placement System.

$X$-ray localization of recording electrode cannulas. At the end of the surgery, a specially designed plastic alignment frame was fitted to each of the two animals with an implanted head fixation post to allow later positioning in stereotaxic coordinates for precise $x$-ray analysis of electrode cannula positions. In brief, the alignment frame was positioned on the stereotaxic instrument while the animal's head was in place, and a clamp was permanently affixed to it, "memorizing" the position of the head in relation to the stereotaxic frame. The clamp in the frame served to realign the animal later in stereotaxic coordinates during $x$-ray localization of the recording electrode cannulas. The position of the clamp in the frame was specific for each animal, so that a framc was used for only one animal at a time.

Postsurgical $x$-ray analysis of cannula positions was very useful in the initial localization of the LC with the recording probes. As shown in Figure 1, the alignment achieved with this device was excellent, and allowed $x$-rays to be taken in an apparent stereotaxic plane. The inner cannulas containing the microwires were easily visualized on such $\mathrm{x}$-ray photos, and the position of these cannulas with respect to the auditory 

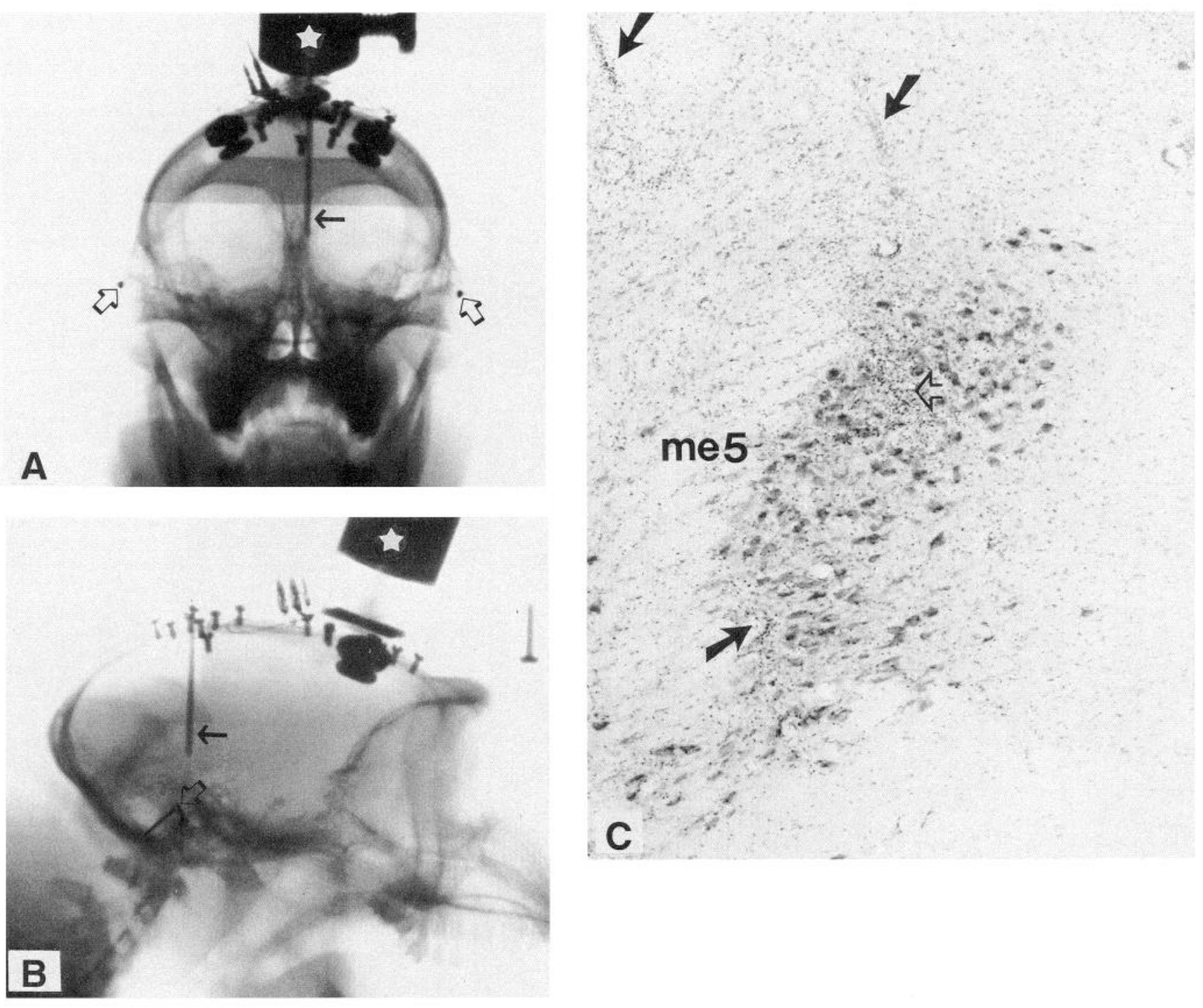

Figure 1. $A$ and $B$, X-ray photographs showing frontal $(A)$ and sagittal $(B)$ views of an experimental monkey's head; these were taken with the plastic alignment frame to place the head in stereotaxic planes. Solid arrows indicate the inner cannulas that hold the recording microwires, which extend about $5 \mathrm{~mm}$ more ventrally (microwires are not visible). Open arrows indicate metal inserts placed into the auditory meatus; note that the auditory meatus are superimposed in the sagittal view $(B)$. The LC is located approximately $1 \mathrm{~mm}$ rostral and $5 \mathrm{~mm}$ dorsal to this point, $2 \mathrm{~mm}$ from the midline. The cannula could be adjusted to direct the inner microwires toward this location. The alignment frame clamp that holds the implanted fixation post is indicated by the stars. $C$, Photomicrograph of a frontal section $(40 \mu \mathrm{m}$ thick $)$ taken through the LC of a monkey used in these experiments, stained with an antibody against tyrosine hydroxylase to identify noradrenergic neurons in the LC (darkly stained cells) and counterstained with cresyl violet. Note glial-filled electrode penetrations aimed at the LC (at solid arrows) and the glial-filled scar marking a recording site in the nucleus of LC neurons (at open arrow). Medial is to the right and dorsal is to the top. Note the close juxtaposition of the tract of the mesencephalic nucleus of the trigeminal nerve (me5) immediately lateral to the LC.

meatus (visualized by inserting blunt steel inserts atraumatically into the external ear cavities) was determined. By adjusting the cannulas from such measurements, initial microwire recordings were obtained from areas very nearby, or within, the LC. This greatly facilitated subsequent adjustments of electrode position, which could be based upon the known electrophysiological characteristics of areas neighboring the LC.

Recording methods. In the animals with permanently implanted bundles of microwires, bundles in each hemisphere were advanced 200$400 \mu \mathrm{m} / \mathrm{d}$ in $40-80 \mu \mathrm{m}$ increments while monitoring each wire for neuronal activity.

For recording neuronal activity from LC neurons in animals with adjustable guide cannula allowing multiple penetrations, one or two microwire electrodes (25- $\mu \mathrm{m}$-diameter stainless steel, Teflon insulated; California Fine Wire, Inc.) with beveled tips were used. These microwires were placed in a custom-designed, precision miniature screwdriven microdrive that was attached to the guide cannula of the LC recording implant. This microdrive advanced the electrodes up to 16 $\mathrm{mm}$ beyond the end of the guide cannula in $20 \mu \mathrm{m}$ steps in waking animals. These electrode movements were made by remote control without disturbing the animal. The depth of penetration was measured with $0.1 \mathrm{~mm}$ accuracy, and this accuracy applied to all penetrations made from the same pedestal. A thin insulating sheath (polyimide tubing) covered each wire bundle for nearly its entire length.

The locations of physiologically identifiable brain structures were carefully noted on each recording penetration to help localize the LC. 
A

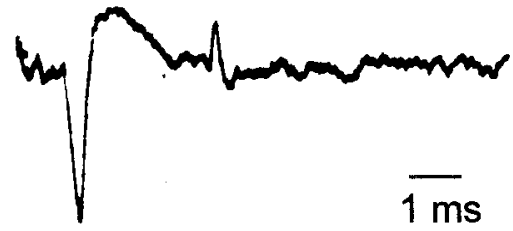

B

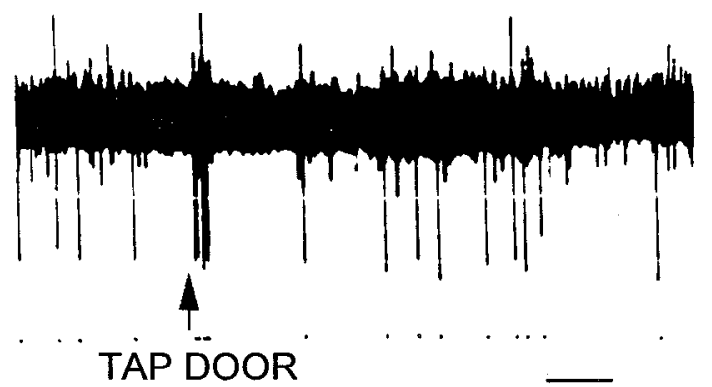

$0.2 \mathrm{sec}$
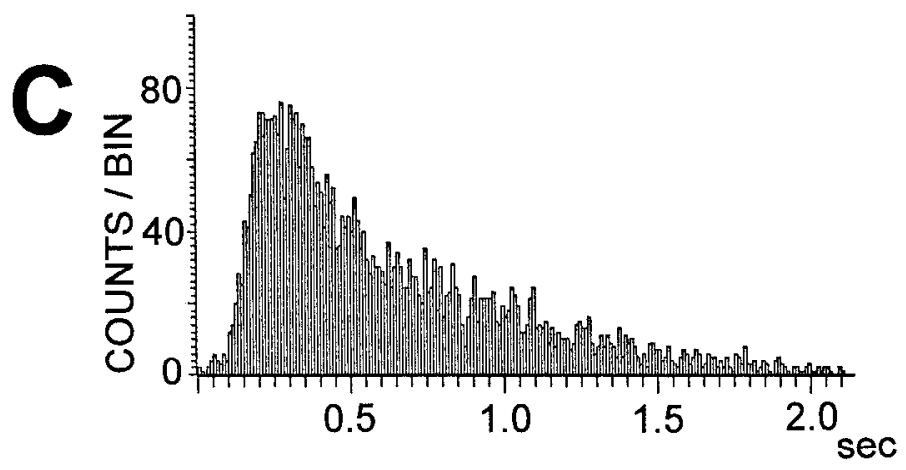

D

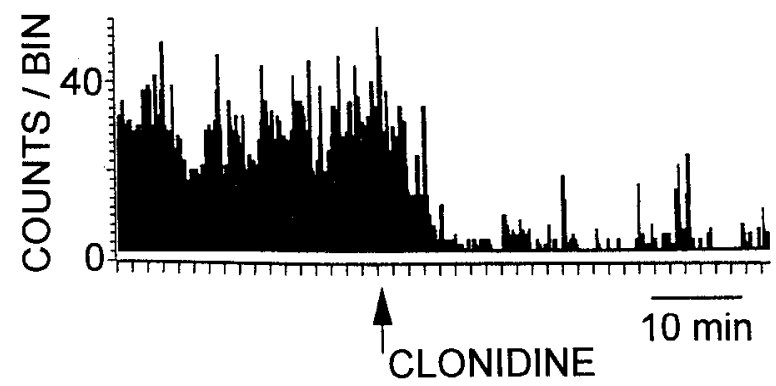

Figure 2. A, Waveform of a typical LC neuron recorded from a monkey during task performance. Note the wide spike (about $2 \mathrm{msec}$ in this case). Negative is downward. $B$, Slow-speed recording from a monkey LC neuron in response to a single tap on the door of the experimental chamber (at arrow). Note the stable spike size and the phasic activation followed by postactivation inhibition, typical of $\mathrm{LC}$ neurons in response to such stimuli that elicit orienting behavior (as in this case). $C$, Inter spike interval histogram of tonic activity for a typical single LC neuron recorded during the task. Note the slow tonic activity, also typical of these neurons recorded in rat (Aston-Jones and Bloom, 1981; Sara and Segal, 1991) and for some cat LC neurons (Chu and Bloom, 1973; Rasmussen et al., 1986). $D$, Time plot showing decrease in tonic LC activity induced by an intramuscular injection of clonidine $(20 \mu \mathrm{g} / \mathrm{kg}$, at arrow). Such reduced activity subsequent to a low dose of clonidine is also characteristic of NE-LC neurons (Svensson et al., 1975).

Structures that were particularly useful landmarks in this regard were the superior and inferior colliculi, the fourth cranial nerve, the cerebellum, and neurons of the mesencephalic nucleus of the trigeminal nerve. Characteristic properties of LC neurons observed in previous studies (Foote et al., 1980; Grant et al., 1988) were used to identify LC neurons tentatively during recording sessions. Only recordings from neurons meeting the following criteria as noradrenergic $\mathrm{LC}$ neurons were accepted for analysis: (1) proper location with respect to readily identifiable landmarks in recordings, including the inferior colliculus, fourth cranial nerve, cerebellum, and the mesencephalic nucleus of the trigeminal nerve; (2) neuronal activity consistent with properties of LC neurons in previous studies in primate, including slow tonic activity, decreased tonic rate with drowsiness, and phasic activation and postactivation inhibitory pause following novel stimuli (Foote et al., 1980; Grant et al., 1988; Aston-Jones et al., 1991) (see Fig. 2); (3) histological localization of the recording site to the area of noradrenergic LC neurons in brain sections (Fig. 1). Recordings were classified as being from an individual neuron if spike amplitudes were at least three times the baseline noise, and spikes could be reliably and stably discriminated to generate interspike interval histograms with no appreciable activity in the early bins (Fig. 2).

Recording stable, isolated neuronal activity from the LC of waking monkeys is technically difficult, owing to the small size of the nucleus, its depth in the brain, and the inherent instability of recordings from deep brain structures located near a ventricle. Several new features of our technique overcame these difficulties. (1) Use of a stereotaxically accurate head holder for postsurgical $x$-ray localization of electrode position was helpful in early penetrations in each animal, to place recording probes rapidly within the immediate vicinity of the LC. (2) Best (highest signal:noise) and most stable recordings were obtained with beveled microwire electrodes. Attempts with conventional etched tungsten microelectrodes yielded acceptable recordings for only a few penetrations per hemisphere, perhaps reflecting damage inflicted by the larger shafts of these probes in the small nucleus LC. (3) Our customdesigned head-mounted microdrive and positioner allowed several penetrations to be made in close juxtaposition without removing the recording microwires. This reduced damage and eliminated the need to realign subsequent penetrations accurately with previous placements. The ability to reposition the electrode precisely and penetrate the same small area repeatedly in each animal yielded substantial information about surrounding structures, and allowed us to use these landmarks to "zero in" accurately and confidently on the small LC nucleus over the course of tens of penetrations.

For signal processing a custom-built multichannel preamplifier assembly was used that attached to a connector on the animal's head. This four- or eight-channel preamplifier allowed for recording from several separate wires (monopolarly or differentially) by remotely switching positions on a control panel, without disturbing the animal.

EEG signals were recorded differentially between two skull locations and were then fed through amplitiers and filtered at $0.15-75 \mathrm{~Hz}$ bandpass. EEG activity on two channels was digitized $(150 \mathrm{~Hz})$ and continuously stored on the same computer disk as unit and other signals (1401 Laboratory Interface, Cambridge Electronic Design Limited, Cambridge, UK, and MS-DOS microcomputer)

An RK-416 infrared viden-based Fye Tracking System (ISCAN, Cambridge, MA) was used to monitor eye position and pupillary diameter continuously during this task in animals with fixed heads. This noninvasive system provided continuous vertical and horizontal eye position and pupil diameter information with $0.5^{\circ}$ spatial accuracy and $16.6 \mathrm{msec}$ temporal resolution.

Window discriminator pulses, analog physiological signals, the ISCAN eye position and pupil diameter signals, and pulses triggered by lever presses, stimulus events, and licking of the fluid spout were fed into the CED 1401 interface and microcomputer for on-line storage and analysis. Data from all experiments were archived on a large-storagecapacity LF-5010 Panasonic WORM optical disk drive.

Behavioral task and experimental protocol. The task employed during recording sessions is illustrated in Figure 3, and was similar to that described above for hehavioral training except with the addition of visual fixation for the two animals with fixed heads. To initiate each experimental trial, each animal was required to depress a lever located near his hand. The two animals with fixed heads also were required to foveate continuously a spot in the center of the monitor (fix spot; $0.3^{\circ}$ of visual angle) for 230 or $550 \mathrm{msec}$. Immediately after depression of the lever and successful foveation of the fix spot, a CS + or CS - stimulus was presented ( $665 \mathrm{msec}$ duration). For testing using the video monitor, cues were presented in place of the fix spot. The animal was required to release the lever within $665 \mathrm{msec}$ of $\mathrm{CS}+$ onset to receive a drop of juice (Tang, approximately $0.3 \mathrm{ml}$ ). Incorrect releases to nontarget stimuli generated a time-out for $3 \mathrm{sec}$ during which responding had no effect. Target cues occurred on $10-50 \%$ of trials (most testing was at $10 \%$ or $20 \%$ ), and were semirandomly dispersed among nontarget stimuli. In- 
horizontal eye position
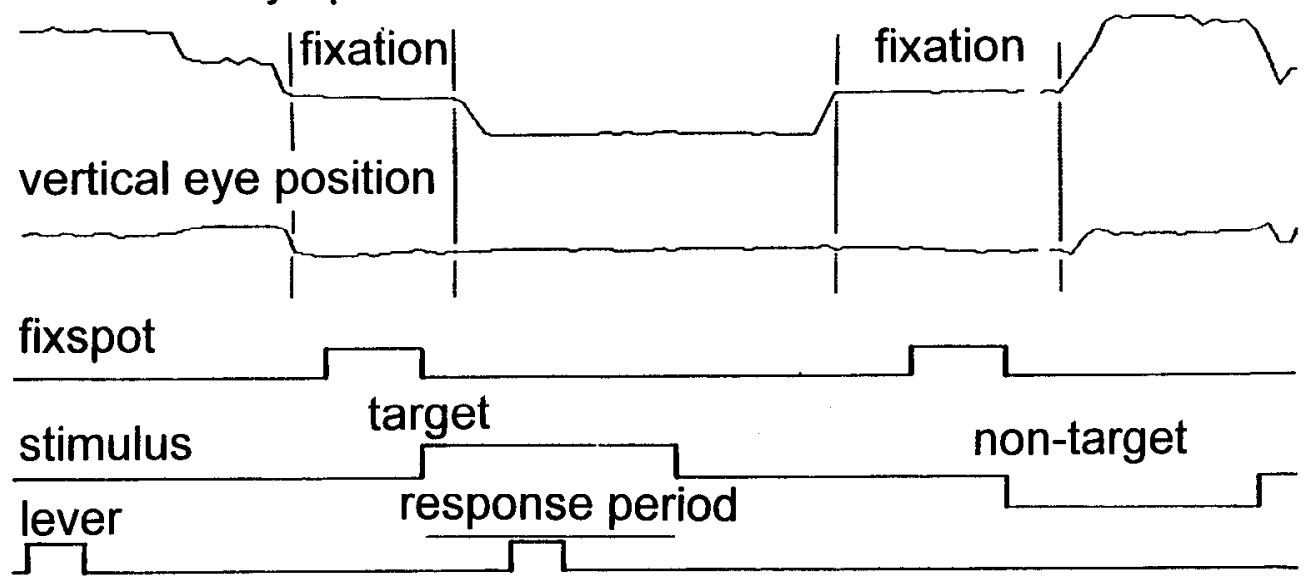

reward 几
Figure 3. Recording of a typical segment of behavior during an experiment, illustrating the task employing line segments on a video monitor as cues. Immediately after the animal depressed the lever, a fix spot appeared in the center of the video monitor. The animal was required to foveate the spot for 230 or $550 \mathrm{msec}$, after which a stimulus cue appeared in place of the spot. The animal had to release the lever within $665 \mathrm{msec}$ of target cue onset to receive a drop of juice reward. Failure to depress the lever prevented fix-spot and cue presentation, and failure to foveate the fix spot $\left( \pm 1^{\circ}\right.$ acceptance window) prevented cue presentation. Lever releases for nontarget cues yielded a 3 sec time-out. Fix spots were presented at an average (but unpredictable, semirandom) interval of $1.7 \mathrm{sec}$.

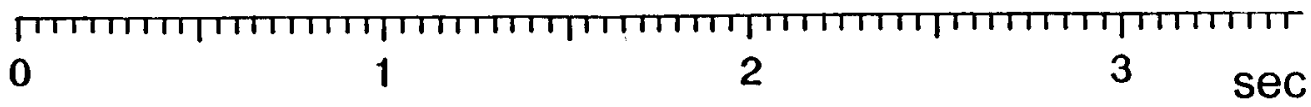

response interval - number of counts expected in this interval in baseline (prestimulus) activity. These response magnitudes were statistically compared using paired or unpaired $t$ tests, as appropriate.

The excitability ratio of LC neurons for target versus nontarget cues was defined as (LC response magnitude for target cues) $\div$ (LC response magnitude for nontarget cues). This was calculated as the ratio of the numbers of spikes over baseline in the response intervals of PSTHs for target versus nontarget cues, each containing 100 trials. The response interval used for each cell was defined from PSTHs for target cues as the poststimulus time between the response onset and offset (as defined above). Because cells did not exhibit significant responses to nontarget cues, the interval used in target cue PSTHs was also used for nontarget cue PSTHs for calculation of the larget:nontarget response ratio.

\section{Results}

\section{Behavioral performance}

Animals learned to perform this task with fewer than $10 \%$ errors after 3-6 months of training. Sessions typically resulted in 15001900 trials per hour, with 140-180 hits, 1400-1600 correct rejections, and 70-150 false alarms during a $1 \mathrm{hr}$ period. Sessions while recording an individual neuron typically lasted 30 90 min, so more than 1000 stimulus trials were accumulated during recordings for most cells. While the overall level of performance was high, it is noteworthy that performance varied markedly for different epochs during a session. Periods of no task performance, or with a high rate of false alarm responses, were intermixed throughout a session with periods of continuous, error-free performance (Fig. 4). Analysis of task behavior in terms of the signal detection parameters $d^{\prime}$ (a measure of ability to discriminate $\mathrm{CS}+$ and $\mathrm{CS}-$ cues) and $\beta$ (a measure of the animal's response criterion; Parasuraman and Davies, 1976; Warm and Jerison, 1984) revealed that epochs of high false alarm activity yielded reduced $d^{\prime}$ and $\beta$ compared to epochs of few false alarms. As shown in Figure 4, these values often fluctuated substantially during a single session. Incorrect omissions were rare on this task, and none were observed in most recording sessions.

Task behavior was arbitrarily divided into two performance categories, denoted as excellent or poor. Periods of excellent
The response magnitude for each PSTH was calculated according to the following equation: response magnitude $=$ number of counts in 

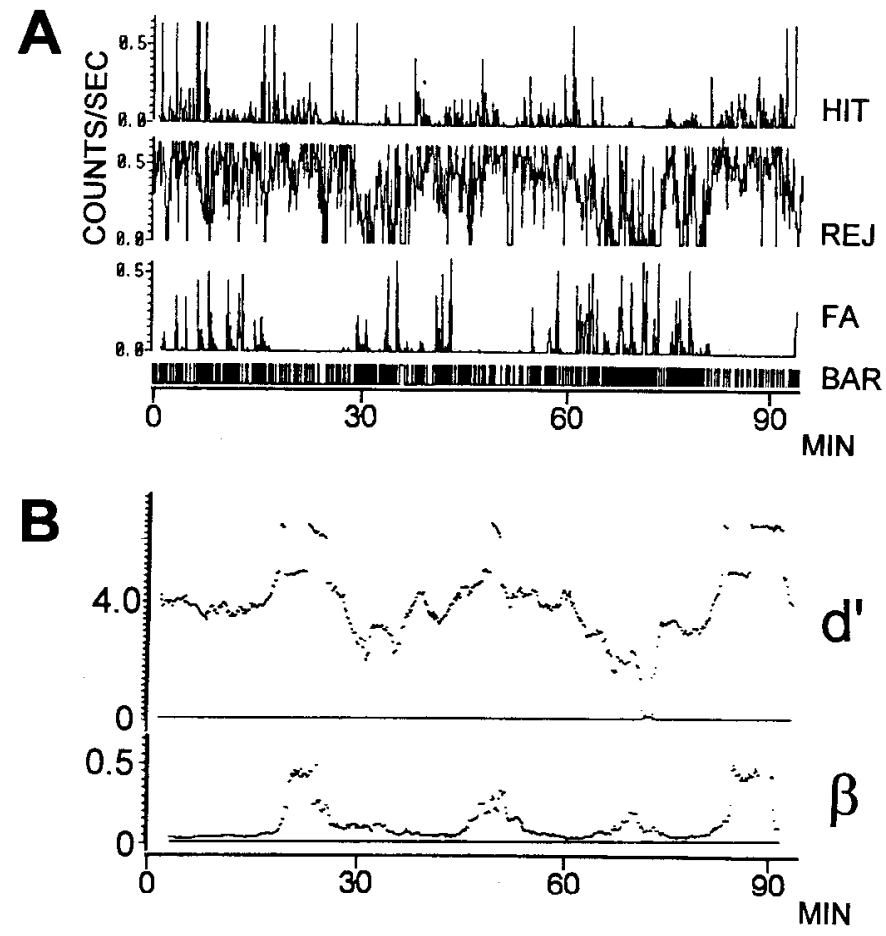

Figure 4. A, Time histogram display of the frequency of various behavioral responses (indicated to the right; HIT, hits; REJ, correct rejections; $F A$, false alarms; $B A R$, lever releases) in a monkey performing the video-based task continuously for more than $90 \mathrm{~min}$. Note the episodic appearance of poor performance (indicated primarily by increased false alarm responding) among periods of error-free performance. Monkeys committed very few incorrect omissions (none in this behavioral segment); the decreased frequency of hits reflects the frequency of target stimulus presentations, which depended upon depression of the lever, foveation of the fix spot, and time-outs generated from false alarm responses. $B$, Continuous plots of the signal detection parameters $d^{\prime}$ and $\beta$ during the same 90 min of a monkey's task performance as in $A$. Note that $d^{\prime}$ and $\beta$ vary substantially during the course of a single session, and that both increase with decreased frequencies of false alarms, indicating improved performance. The $d$ ' parameter was calculated for $200 \mathrm{sec}$ epochs of performance, updated and plotted every $20 \mathrm{sec}$. The $\beta$ parameter was calculated for $400 \mathrm{sec}$ epochs of performance, updated and plotted every $40 \mathrm{sec}$.

behavior were defined as $10 \mathrm{~min}$ or more that contained less than $2 \%$ false alarm responses to $\mathrm{CS}-$ cues, and periods of poor performance were defined as $10 \mathrm{~min}$ or more that contained more than $6 \%$ false alarm responses (range $=6.2-36 \%$; higher rates of false alarm responding were not consistent with maintenance of task performance over 10 min or more). This method of calegorizing the quality of task performance was tested by analyzing another performance parameter, lever-release latency. Results of this analysis supported our criteria for levels of performance, revealing that latencies of lever release were significantly shorter during periods of excellent performance $($ mean $=$ $282 \pm 8 \mathrm{msec}$ ) compared to epochs of poor performance (mean $=306 \pm 13 \mathrm{msec} ; p=0.004$, paired $t$ test).

\section{$L C$ recordings}

Forty-seven individual LC neurons, and 126 instances of multicellular activity, were recorded from the LC of the four monkeys during performance of the task. As shown in Figure 2, impulse waveforms for individual neurons were well discriminated from baseline noise or other neurons, and were stable, so activity could be routinely monitored for over $30 \mathrm{~min}$ ( $\mathrm{min}$ imum acceptable time for inclusion in data pool) to over $3.5 \mathrm{hr}$ (longest attempted). Multiccll activity was studied only if spikes were clearly discriminable with amplitudes greater than twice the baseline noise and remained stable and artifact free throughout the recording session. Such recordings appeared typically to be composed of spikes from more than two neurons, but were not composed of background "hash" as seen with recordings from large populations of neurons. Tonic discharge rates of individual LC neurons during alert task performance ranged approximately from 1.0 to 5.0 spikes/second. Two neurons meeting the criteria for noradrenergic LC cells were recorded after systemic clonidine administration $(20 \mu \mathrm{g} / \mathrm{kg}$, i.m.; injections separated by several days), and both were rapidly and profoundly inhibited by this agent (Fig. 2).

\section{$L C$ responses to task stimuli}

A typical and distinctive feature of LC neurons recorded in all monkeys was that these cells werc sclectively activated in a phasic manner by target cues (CS+ stimuli). All LC neurons examined elicited apparent phasic activation following target cues. The 12 LC neurons yielding high-quality stable recordings for at least $60 \mathrm{~min}$ were subjected to quantitative analysis; this long recording epoch was needed to provide sufficient numbers of trials with target cues to allow confident quantitative analyses. These cells were recorded in the task employing line segments of different orientations as cues, and exhibited properties that were representative of our overall population. When analyzed for 100 target cue trials, nine of these neurons yielded significant excitatory responses, while the remaining three cells exhibited an apparent but nonsignificant phasic activation by our criteria (see Materials and Methods). However, two of these three cells exhibited significant activation when analyzed over larger numbers of target cues $(>200)$.

As shown for a typical cell in Figure 5, LC neurons were phasically activated by target cues with a mean $( \pm$ SEM) onset latency of $108.4 \pm 10.4 \mathrm{msec}$, and a duration of $67.6 \pm 7.0$ msec. These latencies were significantly shorter than the latencies of behavioral responses to target cues, which were $290.2 \pm$ $18.2 \mathrm{msec}$ on average. In contrast to these responses to target cues, nontarget cues elicited no significant activation of these same LC neurons in 100 trials. Similarly, no other LC neuron exhibited any apparent excitation following nontarget stimuli when averaged over 100 trials. Weak excitatory responses to nontarget stimuli were revealed in some neurons only when averaged over a very large number of trials ( $>1000)$.

We calculated the excitability ratio for responses of $\mathrm{LC}$ neurons to target cues versus nontarget cues (described in Materials and Methods) for the nine individual LC neurons that yielded significant responses with 100 target trials (described above). This ratio was 3.6, indicating that $\mathrm{LC}$ responses were 3.6 times greater following target cues than during the same interval following nontarget cues.

As also seen in Figure 5, LC neurons were not substantially affected by fix-spot stimuli or lever-release behavior. Some cells exhibited modestly decreased discharge following presentation of the juice reward, which may correspond to lower activity during liquid consumption, as has been previously reported (Aston-Jones and Bloom, 1981; Grant et al., 1988).

All multicell recordings were subjected to PSTH analysis, and such activity recorded from the LC exhibited properties similar to those of single neurons reported above. Thus, all instances 


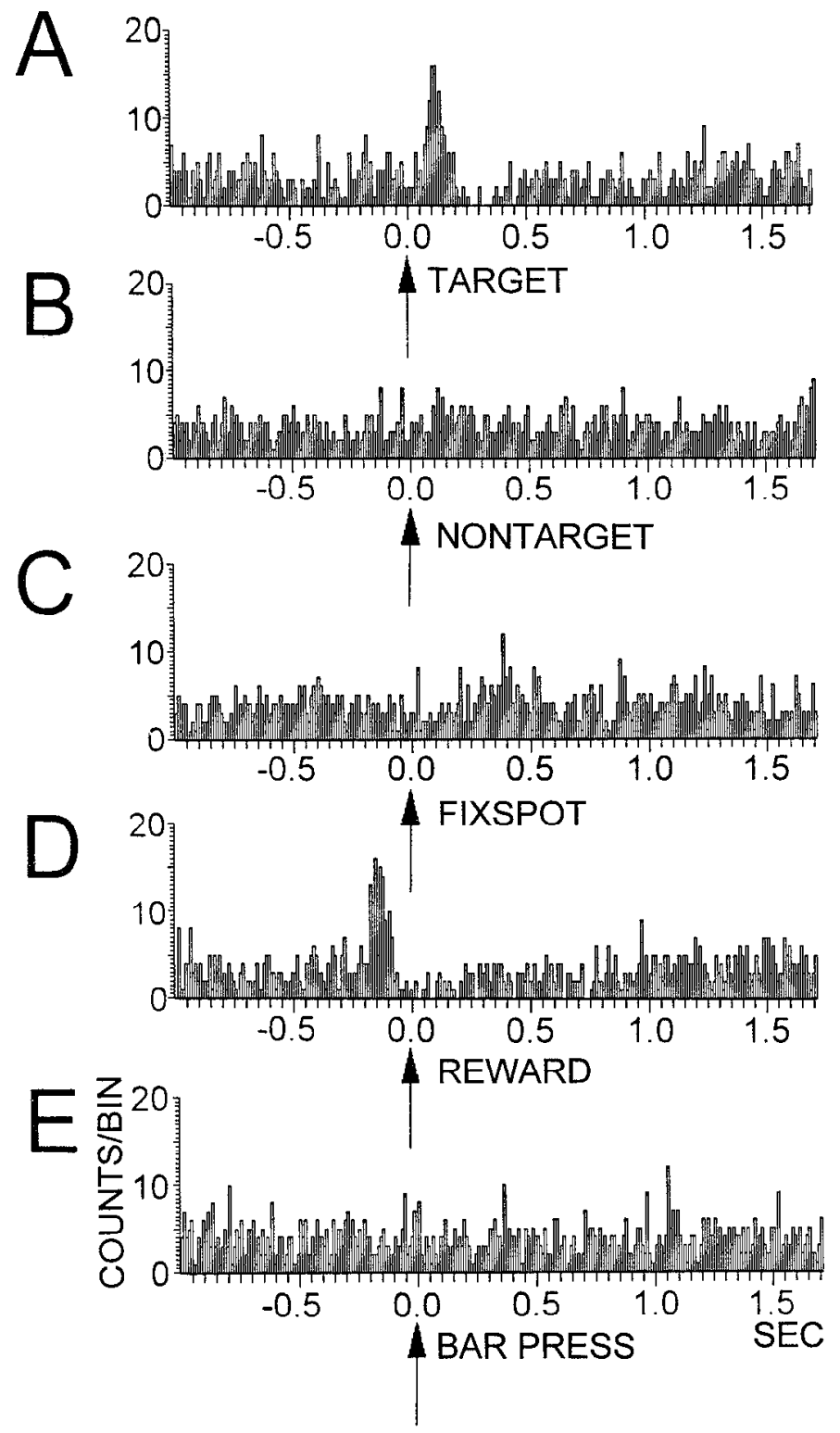

Figure 5. PSTHs for a typical individual LC neuron in response to various events during performance of the vigilance task. PSTHs are each accumulated for 100 sweeps of activity in this neuron synchronized with target stimuli $(A)$, nontargct stimuli $(B)$, fix-spot presentation $(C)$, juice solenoid activation $(D)$, or bar press and release performed outside of the task $(E)$, as indicated. Note the selective activation by target stimuli $(A)$. The small tendency for a response in $C$ may reflect activation by target stimuli that occur at short but variable times after fix spots. Similarly, the activation seen before reward presentation $(D)$ is due to activation by target cues.

of multicell recordings from the LC exhibited selective activation by target cues in the task, and were not consistently affected by other task events. Figure 6 shows PSTHs accumulated for a typical multicell recording in subject BB. Quantitative analysis was conducted for 12 multicell recordings in the $\mathrm{LC}$ that were maintained for at least $60 \mathrm{~min}$ during task performance, and that were representative of our overall multicell sample. The latency of response onset to target cues for these 12 recordings was $90.7+6.0 \mathrm{msec}$, and response duration was $100.7 \pm 6.0$ msec. The onset latency is somewhat shorter, and duration somewhat longer, than for single neuron recordings (above), as

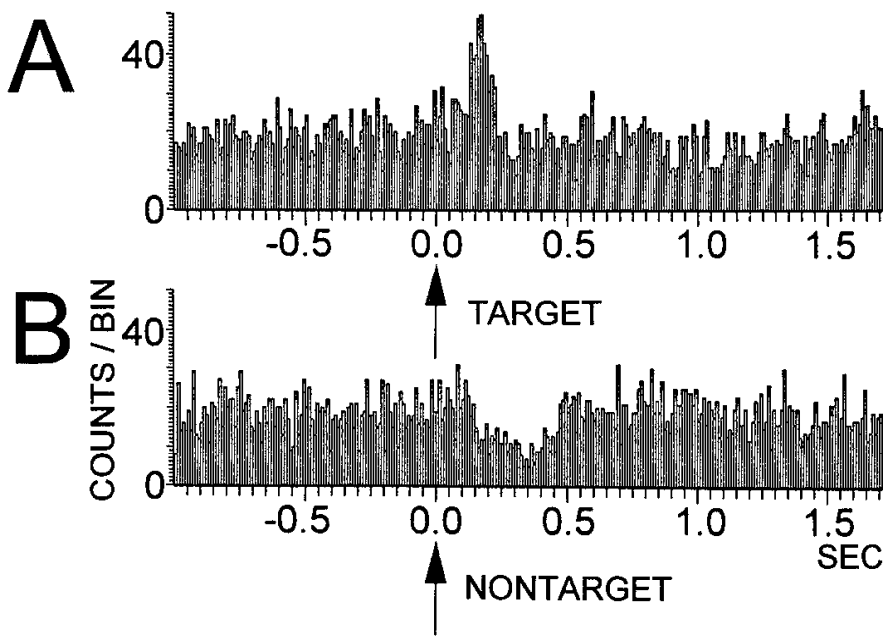

Figure 6. PSTHs for a typical LC multicell recording in subject BB in response to target stimuli $(A)$ or to nontarget stimuli $(B)$. Note that the cell elicited a short-latency phasic activation after target cues, as LC neurons in other monkeys tested. However, in this animal nontarget cues consistently evoked a phasic inhibitory response, as in $B$. Such inhibitory responses were never seen in the other three monkeys tested. Cues were line segments presented on a video monitor, as described in Materials and Methods. Each histogram is composed of 100 trials.

expected given the larger number of spikes sampled per histogram.

In one animal (subject BB), nontarget stimuli yielded phasic inhibition of LC neurons; these same neurons were phasically activated by target cues (Fig. 6). These inhibitory responses to nontarget cues were not seen in any LC neuron of the other three monkeys studied, but occurred in every LC recording in one animal. Inhibitory responses (measured for 100 nontarget stimuli) were $217.6 \pm 23.7 \mathrm{mscc}$ in onset and $230.4 \pm 41.4$ msec in duration.

Three monkeys were subjected to cue reversal, so that the previous target cue became the nontarget cue, while the previous nontarget cue became the rewarded, infrequent target cue. During reversal training, all $\mathrm{LC}$ neurons tested reversed their cueresponse profile and became selectively responsive to the new target cue and unresponsive to the old target (new nontarget) cue (Kubiak et al., 1993; P. Kubiak, J. Rajkowski, and G. AstonJones, unpublished observations). As illustrated for a typical neuron in Figure 7 , the selectivity of LC responses for target cues was maintained regardless of the color or orientation used as target.

\section{Alterations of $L C$ response to target cues as a function of behavioral performance}

Inspection of LC responses over time during the task revealed that response amplitudes to target cues varied periodically, in accordance with the animal's level of behavioral performance. As shown for a typical LC neuron in Figure 8, during periods of poor behavioral performance $\mathrm{LC}$ responses to target cues were smaller in amplitude than during epochs of excellent behavioral performance; most cells failed to respond at all during periods of poor performance. This change in response magnitude with behavioral performance was characteristic of $\mathrm{LC}$ recordings in the two animals tested (cues were lines of different orientation). Neurons that were recorded during substantial epochs of both excellent and poor performance ( $>30 \mathrm{~min}$ each) were subjected 

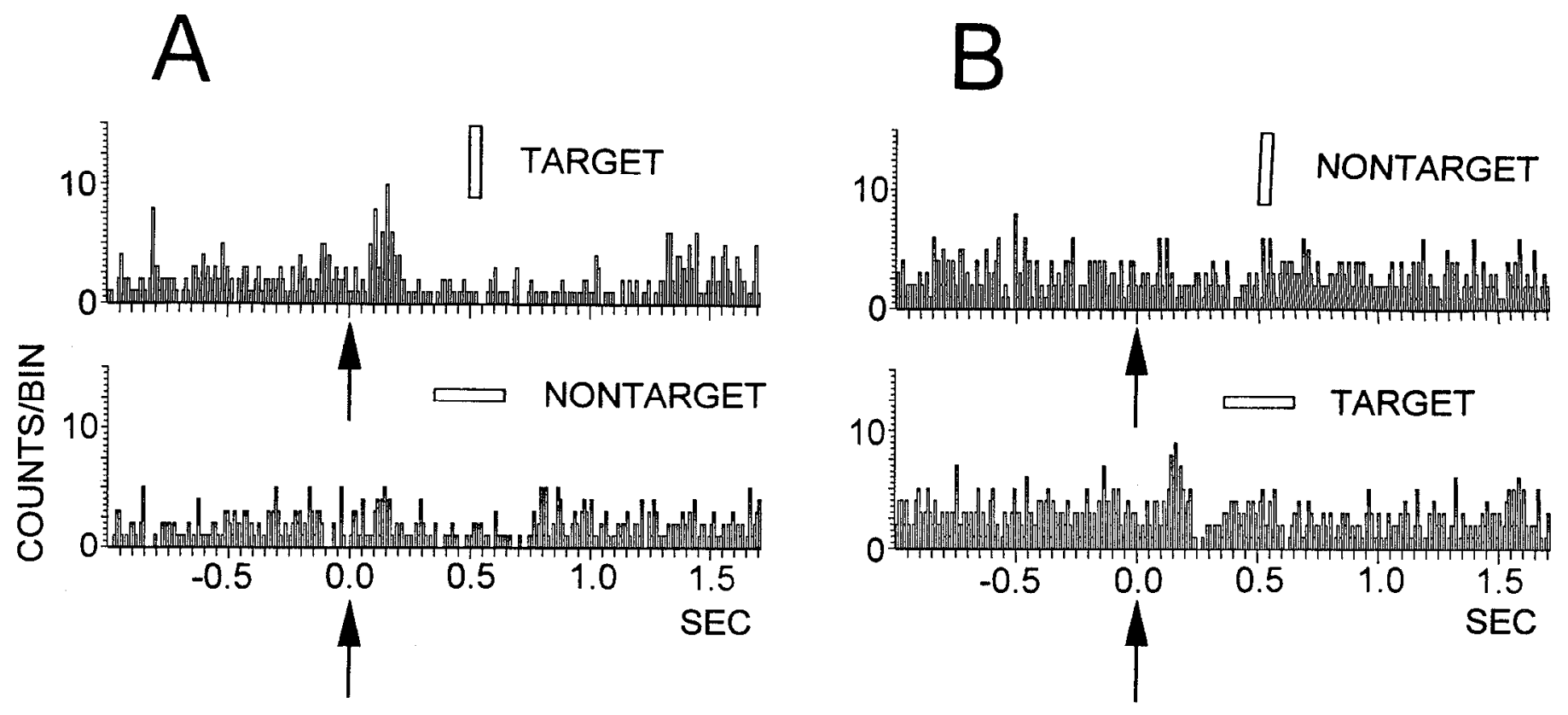

Figure 7. PSTHs for individual LC neurons recorded from the same subject before $(A)$ and after $(B)$ reversal of stimulus meaning. Note that the cell in $A$ was activated by vertical stimuli when they were target cues and not by the nontarget horizontal stimuli. This was typical of all LC neurons recorded during this stimulus contingency. In contrast, after reversal the neuron in $B$ was selectively activated by horizontal stimuli when they were target cues and not activated by the vertical stimuli when they were nontarget cues. This response profile was typical of $L C$ neurons recorded during this stimulus contingency. This indicates that LC neurons respond to the meaning of stimuli, and not to their physical attributes. PSTHs in $A$ were taken for the same LC neuron during one experimental session of intermixed target and nontarget cues; the same procedure was used for a different neuron in $B$, several days after cue reversal in the same monkey. Each PSTH is composed of 100 trials.

to quantitative analysis, which revealed that this difference between $L C$ response magnitudes during poor versus excellent behavioral performance was significant ( $p<0.004 ; n-6$ cells). It is noteworthy that $\mathrm{LC}$ responses to target cues were reduced during periods of poor performance even though these cues always yielded correct behavioral responses (hits).

The relationship between $\mathrm{LC}$ responses and behavioral responses were also analyzed in a trial-by-trial manner. Specifically, the latency of the first spike in an LC neuron following each target cue was compared to the latency of the corresponding behavioral response (lever release). This comparison was restricted to periods of excellent behavioral performance as LC responses to target cues occurred only during such times (described above). This analysis revealed a highly significant positive correlation between $\mathrm{LC}$ response and behavioral response ( $r=0.30 ; p<0.0001 ; n=197$ trials for five cells), indicating that shorter-latency $L C$ responses were associated with shorter behavioral response latencies.

We also tested whether an LC response to a target cue altered the behavioral response to the following sensory cue. For this, we analyzed the rare occasions when the semirandomly presented target cues occurred in pairs, and compared the latency of the lever releases for the first and second cues in each pair. All of these target cue pairs were preceded by a nontarget cue in the stimulus series. Examination of the corresponding PSTHs confirmed that LC neurons were activated by each of the cues in these pairs; moreover, the magnitude of response for the first target cue was significantly greater than that for the second target cue of the pair $(p<0.05)$. For the seven sessions with 33 target cue pairs examined, the lever-response latency for the second target cue $(260 \pm 7 \mathrm{msec})$ was significantly shorter than for the first cue $(283 \pm 8 \mathrm{msec} ; p<0.01$, paired two-tailed $t$ test $)$. This indicates that behavioral responses to target cues that are preceded by a cue-evoked LC response were faster than behavior al responses to the same cues preceded by nontarget stimuli that did not evoke LC responses. We also examined bar-release latencies for target cues that were preceded by a nontarget cue that generated either no response (correct rejection) or an incorrect bar release (false alarm). This analysis revealed that there was no consistent difference between bar-release latencies for target stimuli depending upon whether the preceding trial elicited a bar release (eight sessions analyzed with 63 false alarm target pairs and 1089 correct rejection target pairs). Thus, the shorter bar-release latency for the second cue in the target cue pairs was not simply due to the preceding behavioral response.

LC neuronal responses to nontarget stimuli that elicited false alarm behavioral responses were also analyzed. During periods of poor performance with frequent false alarms, LC neurons were not activated by nontarget stimuli that evoked false alarm behavioral responses (Fig. 8). Thus, none of the 17 cells analyzed exhibited a response to nontarget stimuli that elicited cither a behavioral response (false alarm) or no behavioral response (rejection; as described above). As noted above, the response of LC neurons to target stimuli that elicited lever releases (hits) was also markedly attenuated during periods of poor performance. Thus, LC neurons were relatively unresponsive overall during periods of poor behavioral performance.

In addition to these brief fluctuations in behavior, behavioral performance degraded with prolonged task activity, yielding a vigilance decrement typical of such tasks (Davies and Parasuraman, 1982; Parasuraman, 1984; Warm and Jerison, 1984). Comparison of performance in $30 \mathrm{~min}$ epochs at least $60 \mathrm{~min}$ apart during continuous task behavior revealed several changes in task performance in the later epochs compared to the early 

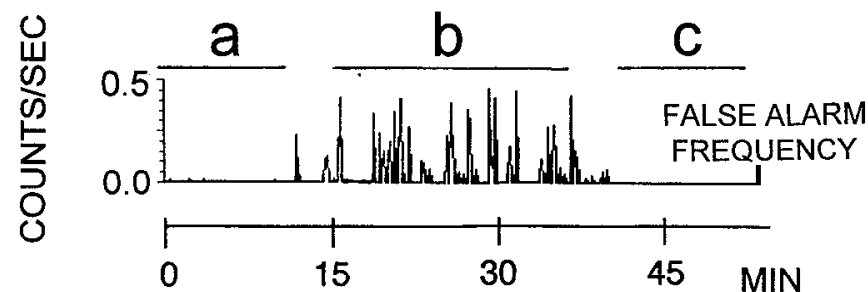

A
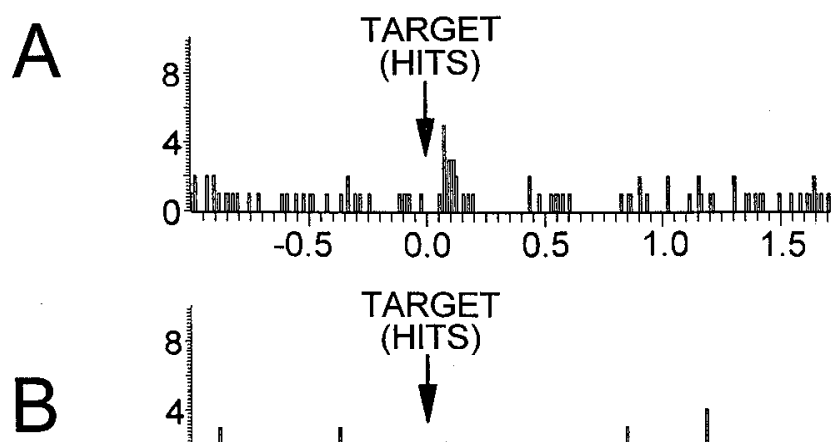

$$
\text { 迹 }
$$
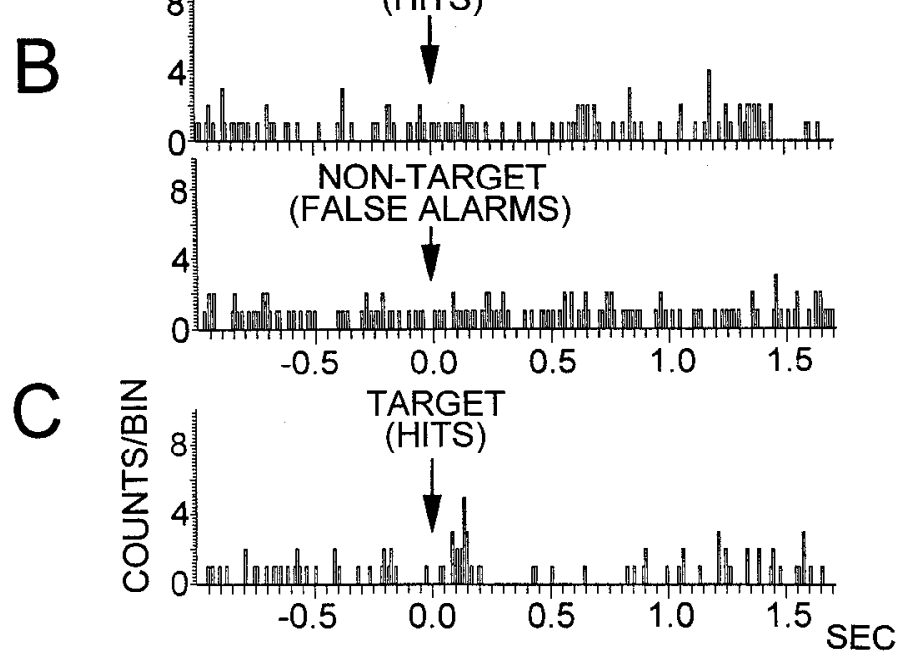

Figure 8. Activity of a typical LC neuron in response to target or nontarget cues during excellent or poor performance. Top. Frequency of false alarm responses during a continuous period of task performance. Periods $a$ and $c$ have very low false alarm rates (excellent performance), while period $b$ has a high frequency of false alarms (poor performance). $A-C$, PSTHs for this LC neuron in response to target or nontarget stimuli during epochs of excellent or poor performance. All stimuli incorporated into these histograms yielded lever releases within the criterion time $(665 \mathrm{msec})$. PSTHs $A-C$ were taken during the corresponding behavioral cpochs $a-c$, respectively, in the top panel, and cach is composcd of 30 sweeps. Note that during the epoch of poor performance, this neuron did not respond to either target or nontarget cues even though these stimuli elicited lever releases. This is in contrast to the phasic excitatory responses apparent during epochs of excellent performance in PSTHs $A$ and $C$. There were insufficient false alarm responses during periods of excellent performance to compare data for these nontarget stimuli with target stimuli that generated hits.

epochs: (1) the frequency of foveating the fix spot decreased substantially, (2) the rate of false alarm responding increased significantly, and (3) the latency of bar release for hits increased significantly, and became more variable (Table 1). Responses of LC neurons also changed significantly between these same time epochs. LC responses to target cues in the later epochs of task performance were significantly smaller than for the same stimuli during the early epochs of task performance (Table 1). Thus, LC responses to target cues decreased significantly in parallel with behavioral performance during a time-dependent vigilance decrement.
Table 1. Measures of behavioral performance and the magnitude of LC response to target cues during early versus late epochs of continuous performance in the task

\begin{tabular}{lclll} 
& \% FAs & Bar-up lat & $\%$ Fix & $R_{\text {mag }}$ \\
\hline Early & 1.2 & $294 \pm 28$ & 76.1 & $34.0 \pm 7.4$ \\
Late & 2.2 & $318 \pm 36$ & 61.2 & $21.7 \pm 7.2$ \\
$P$ value & $<0.02$ & $<0.04$ & $<0.065$ & $<0.01$
\end{tabular}

Early and late epochs were $30 \mathrm{~min}$ in duration, and were separated by 60 min of task performance. Note the correspondence between changes in behavioral measures and LC response magnitudes $\left(R_{\mathrm{mag}}\right)$ from the early to the late task epochs. $\%$ FAs denotes the mean percentage of nontarget cues that elicited false alarm responses; Bar-up lat denotes the mean $( \pm$ SEM) latencies in msec for bar release for hit responses; \% Fix denotes the mean percentage of successful foveations of the fix spot; $R_{\text {mag }}$ denotes the mean $( \pm \mathrm{SEM})$ raw values for response magnitudes of $L C$ neurons to target cues. $P$ values were calculated by $t$ test for bar latencies and response magnitudes, and by the Wilcoxon test for \% FAs and \% Fix. $n=6$ samples for all measures.

\section{Discussion}

Our study revealed that noradrenergic LC neurons in the monkey are phasically activated by target cues during a task designed to measure vigilance, defined as the ability to detect and respond appropriately to infrequent target $(\mathrm{CS}+)$ stimuli embedded among frequent nontarget $\left(\mathrm{CS}^{-}\right)$) cues. These responses are selective for the infrequent target cues regardless of orientation or color; identical stimuli that serve as nontarget cues, or similar stimuli presented outside of the task, generate little or no response in monkey LC neurons. Moreover, the selective response of LC neurons to target cues also occurs following reversal of cue meaning, so that these cells quickly become responsive to the new target cue and stop responding to the previous target cue. Thus, these responses reflect sensitivity to the conditioned attributes or meaning of the cues, not their physical properties. These $\mathrm{LC}$ responses occur at a relatively short latency and precede behavioral responses to the same stimuli by about 200 msec, indicating that such phasic activation of LC neurons is not mediated by feedback from the behavioral response. This is the first report that brain noradrenergic neurons are activated by appetitively conditioned stimuli in an operant task.

We employed several methods and criteria to ensure that recordings were obtaincd from LC ncurons. In chronic expcriments such as ours it is not practical to mark each recording site individually for later histological reconstruction. Instead, we marked the site of a group of recordings that were all obtained from the same small area. We found that LC-like neurons were consistently located within the same small brain region throughout months of recordings, indicating that our penetration technique was very accurate and reliably positioned electrodes within a restricted area. While we cannot rule out possible recordings from non-LC neurons in our study, we conclude that neurons localized to the LC area in this manner and that met our physiological criteria were very likely to be noradrenergic LC neurons.

A noteworthy feature of our results is that different LC neurons exhibited a similar activity profile during the task. This could indicate a recording bias where only the largest cells were discriminated. However, similar results were also obtained for multicell populations containing smaller spikes. Also, this uniformity of physiological properties of LC neurons has been found in nearly all previous studies of these cells (Foote et al., 1983). The similar properties observed here across different LC neu- 
rons support our conclusion that recordings were obtained from NE-LC neurons.

Previous studies have recorded LC neurons during classically conditioned behavior in both cats and rats. In the cat, LC neurons were reported to be activated by $\mathrm{CS}+$ stimuli conditioned by aversive stimuli (air puff) in a conditioned emotional response paradigm. However, in contrast to the present findings, cat LC neurons were not affected by similar stimuli conditioned appetitively with food presentation (Rasmussen and Jacobs, 1986). One reason for this difference may be the species used. In particular, LC neurons in the cat are neurochemically diverse with NE and non-NE neurons closely interdigitated, so it is more difficult to ascribe recordings confidently to NE neurons. Alternatively, the appetitive paradigm used in the cat study was not operant, and did not require a rapid response from the animal to procure food reward. A previous study in rat (Sara and Segal, 1991) reported that LC neurons were activated by CS + cues in both appetitive and aversive classical conditioning paradigms, consistent with the present findings. However, this previous rat study reported that $\mathrm{LC}$ responses rapidly habituated during a constant reinforcement schedule. While responses of LC neurons in the monkey may have decreased over the first few stimulus presentations during training (when no recordings were obtained), it is clear that these cells maintained substantial and selective responsiveness to the target cues after thousands of trials. A possible source of differences between the rat study and the present findings may reflect the nonoperant paradigm that did not require attention in the rat experiment. Thus, comparison of the present findings with previous results indicates that LC neurons may be best engaged by the attentional demands of an operantly conditioned instrumental task.

Paradigms similar to ours have been used extensively in human psychophysical experiments to study vigilance and attention in human performance (reviewed in Davies and Parasuraman, 1982; Warm and Jerison, 1984). Such studies revealed that the ability to detect and respond to rare, unpredictable events varies with a number of factors, most notably time (Parasuraman, 1984). This temporal characteristic is important in distinguishing vigilance or sustained attention from selective attention. In the latter, the intensity of attention, or alertness, is assumed to remain constant, and differences in response among trials are sought as a function of different instructions given for different trials (e.g., attend right vs left; Posner, 1980; Johnston and Dark, 1986). Vigilance, synonymous here as elsewhere with sustained attention, differs from selective attention in that the critical independent variable examined is time rather than instruction set (Jerison, 1977; Parasuraman, 1984; Warm and Jerison, 1984). That is, in vigilance performance the object of the task, and relevant cues, remains the same throughout a typical session. The question of interest is how performance varies as a function of repctition or timc, which is measured as the ability to detect and respond to (sustain attention for) rare, unpredictable events. There is a close interaction between selective attention and vigilance or sustained attention, in that selective attention tasks require high vigilance for performance (Posner, 1992, 1993). Similarly, vigilance tasks typically employ a selective attention component to measure changes in attention over time (e.g., in our lask, larget vs nontarget cue discrimination requires selective attention). Vigilance represents the intensity and duration of attention, and is similar in many regards to the notion of alertness (Posner, 1992, 1993). In fact, alertness could be considered to be synonymous with vigilance where little or no selective attention is involved. In this view, alertness corresponds to nondirected or diffuse vigilance with no specified sensory target, while selective attention represents vigilance with preset sensory targets that trigger specific behavioral responses. Whereas selective attention requires vigilance (as does any other cognitive process), there may be distinct categories of attention. Recent work by Posner and colleagues (Posner and Petersen, 1990; Posner, 1993) indicates that selective and sustained attention may utilize separate brain circuits that interact in behavioral performance.

The present study is the first to indicate that nonhuman primates can be trained to perform classical vigilance tasks, and that their behavior in such a paradigm resembles that of humans (see also preliminary reports of these results; Alexinsky et al., 1990; Aston-Jones et al., 1991). In particular, monkeys performing the present task exhibited periodic epochs of high false alarm rates and a gradual decline in overall performance with prolonged task activity, resembling the vigilance deficit consistently found in human studies (Mackworth, 1970; Parasuraman, 1984; Warm and Jerison, 1984). The stress associated with our experimental situation (chair restraint, head fixation, artificial environment) may have influenced performance, as stress has been found to influence human vigilance performance (Mackworth, 1957; Broadbent and Gregory, 1965; Hockey, 1970; Parasuraman, 1984; Warm and Jerison, 1984; Hancock and Warm, 1989). However, animals were habituated to the chair, experimental chamber, and task over months, so that acute stressors were minimized. The monkeys did not find the task aversive, as they did not resist (and even assisted with) the daily ritual of chairing, and they typically initiated performance immediately after stimuli were available. The ability of monkeys to perform this task has substantial potential for neurobiological experiments to elucidate the brain circuits involved in sustained attention and vigilance.

In our task, target cues (1) were infrequent, (2) indicated the availability of reward, and (3) called for a rapid behavioral response; the phasic activation of $\mathrm{I} C$. neurons by target cues could reflect any of these stimulus attributes. Previous studies of LC activity in several species have revealed that these neurons are activated by unpredicted or novel stimuli (Foote et al., 1980; Aston-Jones and Bloom, 1981; Rasmussen et al., 1986; Grant et al., 1988; Sara and Segal, 1991). These results indicate that the $\mathrm{LC}$ response to target cues in our task may be linked in part to their low probability of occurrence. Consistent with this hypothesis, our preliminary results indicate that the response of LC neurons to target stimuli is lower when their frequency of occurrence is $50 \%$ than when it is 10\% (Alexinsky et al., 1990). However, previous studies also found that LC responses to unconditioned stimuli were substantially greater for those stimuli that elicited an orienting behavioral response (Foote et al., 1980; Aston-Jones and Bloom, 1981; Grant et al., 1988). In vicw of this finding, one might expect stimuli that engage a rapid behavioral response (such as target cues in our task) to activate LC cells. Again our preliminary studies support this possibility, as responses to target cues are larger than those to nontarget cues when the two are equally frequent (50\%; Alexinsky et al., 1990). The possibility that the phasic activation of LC by target cues refiects availability of reward seems unlikely, as LC neurons were not activated by the unconditioned presentation of reward (or the preceding sound of solenoid activation), or the consumption of the juice (as also previously reported; Aston-Jones and Bloom, 1981; Grant et al., 1988). Thus, we propose that 
the phasic responses of LC neurons by target stimuli may reveal that these cells are selectively activated by unpredicted stimuli that demand an immediatc bchavioral responsc. This vicw cxtends previous findings that LC neurons in many species are activated by neutral (unconditioned) stimuli. In the cat and monkey, these responses are pronounced only for the first few occurrences of such stimuli if they are of moderate or low intensity. However, in all species LC phasic sensory responses are associated with stimuli that elicit behavioral activation-for novel unconditioned stimuli this takes the form of an orienting response, while for target stimuli in the present study this takes the form of a rapid lever release.

The response of individual $\mathrm{LC}$ neurons to target cues was small in absolute magnitude, averaging about 0.3 spikes per stimulus (mainly due to the low spike rate of LC neurons overall). However, this relatively small change in spike rate may be functionally significant due to the high uniformity among LC neurons in their response to target cues. Thus, the above analysis combined with the uniformity of $L C$ responsiveness indicates that on any given trial about one-third of LC neurons would elicit an additional spike following a target cue. The widespread efferent arbors of LC neurons, and the associated enormous number of terminal boutons (Moore and Bloom, 1979), may convey a substantial functional impact from this change in spike activity.

Previous studies employing visual tasks with a lever or button response in monkeys revealed that the earliest latency for electromyogram (EMG) activation in major arm muscles after target cues was about $170 \mathrm{msec}$ (Evarts, 1966, 1974), and mean $E M G$ latency $=224 \mathrm{msec}$ (Kimura, 1990). Moreover, the earliest responses of postcentral neurons in monkey with such a task were about 120-150 msec after the target cue (Evarts, 1966, 1972, 1974). As these latencies were obtained in a simple reaction time task not requiring discrimination by the monkey, they are probably shorter than those in our task; indeed, reaction times in those previous studies were on the order of $220 \mathrm{msec}$ (Evarts, 1966) compared to the $290 \mathrm{msec}$ response time in our task (similar to previous values with complex tasks; Nelson et al., 1990). These results indicate that $\mathrm{LC}$ responses to target cues (mean onset latency of $90.7 \mathrm{msec}$ ) substantially precede EMG and postcentral neuronal activation, and that the $\mathrm{LC}$ responses are therefore not a product of activity in primary motor circuits or of sensory feedback from movement. It is also noteworthy that previous reports, like the present study, indicate that the $\mathrm{LC}$ responses are not related to motor activity per se.

Moreover, the latencies at which LC neurons were activated selectively by target cues are among the shortest attention-related responses for sensory and associative brain areas that have been studied extensively with attention tasks. Neurons in the superior colliculus (Goldberg and Wurtz, 1972; Wurtz and Mohler, 1976), pulvinar (Petersen et al., 1985), frontal eye fields (Wurtz and Mohler, 1976; Goldberg and Bushnell, 1981), and striate as well as prestriate cortex (Wurtz and Mohler, 1976; Robinson et al., 1980) exhibit enhanced responses to visual stimuli that are the object of a future saccade. Although the latencies of the enhanced component of responses (which are presumed to reflect attentional attributes) were not specifically calculated in all of these previous studies, inspection of the data indicates that the shortest latencies for enhanced responses were similar to those found here for I.C responses to target cues (with the possible exception of the superior colliculus, which may have somewhat shorter latencies). Other studies of neuronal discharge in the frontal or parietal cortices have found neuronal responses to be selectively enhanced for operantly conditioned, attended stimuli that are not associated with eye movements, more similar to the cues used in our task. The shortest latencies of enhanced responses in these studies were on the order of 80-100 msec (Kubota et al., 1974; Bushnell et al., 1981; Hillyard and Kutas, 1983; Thorpe et al., 1983; Funahashi et al., 1989; Goldberg et al., 1990; B. Desimone, personal communication). Longer latencies have been reported for other structures (e.g., $>200$ msec for inferotemporal cortex, Fuster, 1990; and $>150 \mathrm{msec}$ for hippocampus, Miyashita et al., 1989). One important difference between these experiments and our own is that in all of those previous studies attentional effects produced an enhancement of a preexisting response to visual stimuli; in the LC the response appears to depend more completely on the attentional attributes of the target cue. Despite the differences between these studies and our own, comparison with these previous findings indicates that the LC response may be among the earliest changes in brain that reflect attentional processes. This and the widespread projections of LC-NE neurons also indicate that the LC system may have a role in modulating a wide range of brain activity in accordance with attention.

It is noteworthy that LC impulses evoked by target cues would be expected to reach the cerebral cortex at about the time that certain cortical slow-wave activity is generated, in particular, P3 (similar to P300) waves. These cortical responses have been proposed to reflect late-stage processing of several stimulus attributes including stimulus significance and task relevance (Pritchard, 1981; Donchin et al., 1986). In other of our studies (Alexinsky et al., 1990; Aston-Jones et al., 1991; unpublished observations) we have observed prominent P3-like waves evoked selectively by target cues that also selectively elicit phasic LC responses. Furthermore, studies by Pineda et al. (1989) revealed that P3-like responses in monkey cortex were reduced in amplitude following lesions of the LC. The possibility that the LC is involved in generating these cortical slow-wave events is consistent with the above analysis indicating that $\mathrm{LC}$ activation by a target cue may be part of an attentional process that facilitates the response to that stimulus.

Our results are consistent with a role for responses of LC neurons to target cues in either selective attention processes, or vigilance processes. A role in selective attention might be indicated if the selective LC response to a target cue facilitated the behavioral response to that same cue. Certain of the present results indicate that this is possible. (1) The latency of LC activation by target cues summed with the time for LC impulse conduction to cerebral cortex in the monkey (60-100 msec; Aston-Jones et al., 1985) is less than the minimum behavioral response latency to target cues $(290 \mathrm{msec}$ on average; also see above). (2) This possibility is further supported by our trial-bytrial analysis revealing a positive correlation between the latencies of $\mathrm{LC}$ responses and behavioral responses to target cues. However, the fact that false alarm behavioral responses occur in the absence of $\mathrm{LC}$ responses indicates that $\mathrm{LC}$ activation is not a necessary event in driving a behavioral response to a cue stimulus. It is also noteworthy that stimulus-evoked LC activity cannot play a role in inhibiting behavioral responses to the same stimuli, as nontarget cues that elicit no behavioral response do not activate $\mathrm{LC}$ neurons.

The foregoing analysis indicates that the I C. may play a role in selective attention, as LC responses are selective for target cues and appear to facilitate behavioral responses to the cues 
that elicited LC responses. However, phasic LC responses also occur for nonattended stimuli that are salient by virtue of novelty or intensity (as discussed in the introductory remarks), so not all phasic $\mathrm{LC}$ responses are linked to selective attention processes. If $\mathrm{LC}$ responses facilitate behavioral responses to subsequent cues (e.g., by maintaining task context), then a role in vigilance or sustained attention would be implied. Such a role is consistent with our finding that bar-release latencies were shorter for the second of a pair of target cues (both cues elicited $\mathrm{LC}$ responses in PSIHs). In contrast, bar-release latencies did not differ between target cues that were preceded by a nontarget trial with a bar relcase (falsc alarm) and those that werc preceded by a correct nontarget trial with no bar release (neither nontarget trial is associated with an LC response). This indicates that the facilitated behavioral response for the second of a pair of target cues was not due simply to the preceding behavioral response. While the mechanism for this augmented behavioral responsiveness is uncertain, it is possible that the LC response to the first cue facilitated the behavioral response to the second cue. In this framework, phasic LC responses may also facilitate withholding responses to subsequent nontarget cues. Consistent with this possibility, we found that target-evoked responses of LC neurons were small or absent during epochs of frequent false alarm behavior, and that robust LC responsiveness to target cues was consistently associated with epochs of excellent performance containing few or no false alarms. This may indicate that $\mathrm{LC}$ responses to target cues in our task facilitated selective bchavioral responding to subsequent cues, so that after a phasic $\mathrm{LC}$ response the following nontarget cues are less likely to be erroneously perceived as targets. This could occur if the phasic $\mathrm{LC}$ response increased overall attentiveness to the task (i.e., maintained task context) for several seconds.

Signal detection analysis revealed that during epochs of high false alarm behavior the monkey less effectively discriminated between target and nontarget cues ( $d^{\prime}$ factor was lower), and that the tendency of the animal to respond to any cue increased $(\beta$ factor was lower). The variation in $\mathrm{LC}$ responses to target cues during these same periods is consistent with the possibility that $\mathrm{LC}$ responses contribute to these quantitative measures of vigilance performance by modulating behavioral responses to immediately preceding or succeeding target cues, as described above. Other of our results reveal that the level of tonic LC discharge also varies with behavior on this task, being increased during epochs of poor performance (Rajkowski et al., 1992, 1993; unpublished observations). Thus, tonic or transient changes in LC activity may influence vigilance performance.

At present, our evidence for LC function in either selective attention or vigilance processes is correlative, and cannot establish whether, and to what extent, changes in the LC cause changes in performance. Direct manipulations of LC activity and measurement of behavioral effects in this task will be needed to test this possibility, and to clarify the causal role of the LC system in attention and vigilance.

\section{Conclusions}

Previous results reveal that LC neurons are phasically activated by novel or conspicuous sensory events. The present results indicate that events that are meaningful by virtue of conditioning are also able to activate LC cells phasically. The activation appears to be independent of physical sensory attributes. These findings indicate that cognitive rather than sensory attributes of stimuli may be primarily responsible for evoked activation of LC neurons.

The present results lead us to propose that phasic responses of LC neurons to unpredicted sensory stimuli facilitate adaptive behavioral responses. The global effect of LC activity on neuronal processing may be involved in determining the significance of stimuli, the urgency of response to stimuli, the type of response required, or all of these. One possibility under consideration is that robust phasic LC responses promote rapid, highly determined behavioral responses that require little integration, supplanting complex analysis and more refined behaviors. Conversely, phasic responses of $\mathrm{LC}$ ncurons may facilitate late stages of sensory processing involved in decision making and selecting an adaptive behavioral response. Phasic LC activity may also alter the cognitive context in which subsequent sensory stimuli are received and processed. The magnitude of LC activity, and the behavioral context, may be important in determining which of these functions are conveyed.

The present results have implications for afferent connections to LC neurons. Either LC neurons perform complex integration of inputs from many areas to compute stimulus significance, or the circuits projecting to the LC perform such computations. Areas previously found to exhibit relatively short-latency selective or enhanced responses to attended stimuli include frontal, parietal, and occipital cortices (reviewed above). Previous studies of afferents to the LC in the primate have been limited, but one indicates that the prefrontal cortex projects to the periLC regions containing LC dendrites (Arnsten and Goldman, 1984). Thus, it is possible that cortical areas convey highly processed information to the LC to yield the presently observed selective responses to target cues. However, previous work in the rat indicates that the nucleus $\mathrm{LC}$ receives major inputs from two areas in the rostral medulla, the nucleus paragigantocellularis and the nucleus prepositus hypoglossi, and only limited direct input from other areas (Aston-Jones et al., 1986, 1991). Clearly, anatomical studies are needed in the monkey to determine if afferent connections to the LC in this species resemble those in rat, or include other areas that might be related to the present results.

\section{References}

Alexinsky T, Aston-Jones G, Rajkowski J, Revay RS (1990) Physiological correlates of adaptive behavior in a visual discrimination task in monkeys. Soc Neurosci Abstr 16:164.

Arnsten AF, Goldman RP (1984) Selective prefrontal cortical projections to the region of the locus coeruleus and raphe nuclei in the rhesus monkey. Brain Res 306:9-18.

Aston-Jones G (1985) Behavioral functions of locus coeruleus derived from cellular attributes. Physiol Psychol 13:118-126.

Aston-Jones G, Bloom FE (1981a) Activity of norepinephrine-containing locus coeruleus neurons in behaving rats anticipates fluctuations in the sleep-waking cycle. J Neurosci 1:876-886.

Aston-Jones G, Bloom FE (1981b) Norepinephrine-containing locus coeruleus neurons in behaving rats exhibit pronounced responses to nonl-noxious environmental stimuli. J Neurosci 1:887-900.

Aston-Jones G, Foote SL, Bloom FE (1984) Anatomy and physiology of locus coeruleus neurons: functional implications. In: Frontiers of clinical neuroscience, Vol 2, Norepinephrine (Ziegler M, Lake CR, eds), pp 92-116. Baltimore: Williams and Wilkins.

Aston-Jones G, Foote SL, Segal M (1985) Impulse conduction properties of noradrenergic locus coeruleus axons projecting to monkey cerebrocortex. Neuroscience 15:765-777.

Aston-Jones G, Ennis M, Pieribone VA, Nickell WT, Shipley MT (1986) The brain nucleus locus coeruleus: restricted afferent control of a broad efferent network. Science 234:734-737. 
Aston-Jones G, Shipley MT, Chouvet G, Ennis M, Van Bockstaele EJ, Pieribone V, Shiekhattar R, Akaoka H, Drolet G, Astier B, Charlety P, Valentino R, Williams JT (1991a) Afferent regulation of locus coeruleus neurons: anatomy, physiology and pharmacology. Prog Brain Res 88:47-75.

Aston-Jones $G$, Chiang $C$, Alexinsky T (199lb) Discharge of noradrenergic locus coeruleus neurons in behaving rats and monkeys suggests a role in vigilance. Prog Brain Res 88:501-520.

Broadbent DE, Gregory M (1965) Effects of noise and of signal rate upon vigilance analyzed by means of decision theory. Hum Factors $7: 155-162$.

Bushnell MC, Goldberg ME, Robinson DL (1981) Behavioral enhancement of visual responses in monkey cerebral cortex. I. Modulation in posterior parietal cortex related to selective visual attention. J Neurophysiol 46:755-772.

Chu NS, Bloom FE (1973) Norepinephrine-containing neurons: changes in spontaneous discharge patterns during sleeping and waking. Science 179:908-910.

Crow TJ, Wendlandt S (1976) Impaired acquisition of a passive avoidance response after lesions induced in the locus coeruleus by $6-\mathrm{OH}-$ dopamine. Nature 259:42-44.

Dahlstrom A, Fuxe K (1964) Evidence for the existence of monoamine-containing neurons in the central nervous system. I. Demonstration of monoamines in the cell bodies of brain stem neurons. Acta Physiol Scand 62:5-55.

Davies DR, Parasuraman R (1982) The psychology of vigilance. London: Academic.

Donchin E, Karis D, Bashore TR, Coles MGH, Gratton G (1986) Cognitive psychophysiology and human information processing. In: Psychophysiology: systems, processes and application (Coles MGH, Donchin E, Porges SW, eds). New York: Guilford.

Evarts EV (1966) Pyramidal tract activity associated with a conditioned hand movement in the monkey. J Neurophysiol 29:10111027

Evarts EV (1972) Contrasts between activity of precentral and postcentral neurons of cerebral cortex during movement in the monkey. Brain Res 40:25-31.

Evarts EV (1974) Precentral and postcentral cortical activity in association with visually triggered movement. J Neurophysiol 37:373381.

Everitt BJ, Robbins TW, Gaskin M, Fray PJ (1983) The effects of lesions to ascending noradrenergic neurons on discrimination learning and performance in the rat. Neuroscience 10:397-410.

Foote SL, Aston-Jones G, Bloom FE (1980) Impulse activity of locus coeruleus neurons in awake rats and monkeys is a function of sensory stimulation and arousal. Proc Natl Acad Sci USA 77:3033-3037.

Foote SL, Bloom FE, Aston-Jones G (1983) Nucleus locus ceruleus: new evidence of anatomical and physiological specificity. Physiol Rev 63:844-914.

Freedman R, Foote SL, Bloom FE (1975) Histochemical characterization of a neocortical projection of the nuclcus locus cocrulcus in the squirrel monkey. J Comp Neurol 164:209-231.

Funahashi S, Bruce CJ, Goldman-Rakic PS (1989) Mnemonic coding of visual space in the monkey's dorsolateral prefrontal cortex. J Neurophysiol 61:331-349.

Fuster JM (1990) Inferotemporal units in selective visual attention and short-term memory. J Neurophysiol 64:681-697.

Garver DL, Sladek JJR (1976) Monamine distribution in primate brain. II. Brain stem catecholaminergic pathways in Macaca speciosa (arctoides). Brain Res 103:176-182.

Goldberg ME, Bushnell C (1981) Behavioral enhancement of visual responses in monkey cerebral cortex. II. Modulation in frontal eye fields specifically related to saccades. J Neurophysiol 46:773-778.

Goldberg ME, Wurtz RH (1972) Activity of superior colliculus in behaving monkey. II. Effect of attention on neuronal responses. J Neurophysiol 35:560-574.

Goldberg ME, Colby CL, Duhamel J-R (1990) The representation of visuomotor space in the parietal lobe of the monkey. Cold Spring Harbor Symp Quant Biol 55:729-739.

Grant SJ, Aston-Jones G, Redmond DE Jr (1988) Responses of primate locus coeruleus neurons to simple and complex sensory stimuli. Brain Res Bull 21:401-410.

Hancock PA, Warm JS (1989) A dynamic model of stress and sustained attention. Hum Factors 31:519-537.

Harris GC, Fitzgerald RD (1991) Locus coeruleus involvement in the learning of classically conditioned bradycardia. J Neurosci 11:23142320.

Hillyard SA, Kutas M (1983) Electrophysiology of cognitive processing. Annu Rev Psychol 34:32-61.

Hockey GRJ (1970) Effect of loud noise on attentional selectivity. Q J Exp Psychol 22:28-36.

Jerison HJ (1977) Vigilance: biology, psychology, theory, and practice. In: Vigilance-theory, operational performance, and physiological correlates (Mackic RR, cd), pp 27-40. Ncw York: Plcnum.

Johnston WA, Dark VJ (1986) Selective attention. Annu Rev Psychol 37:43-75.

Jouvet M (1969) Biogenic amines and the states of sleep. Science 163: $32-41$.

Kimura M (1990) Behaviorally contingent property of movementrelated activity of the primate putamen. J Neurophysiol 63:12771296.

Kubiak P, Rajkowski J, Aston-Jones G (1993) Responses of monkey locus coeruleus (LC) neurons to $\mathrm{CS}+$ stimuli reflect cognitive processing: delayed activity during acquisition in a vigilance task. Soc Neurosci Abstr 19:974

Kubota K, Iwamoto T, Suzuki H (1974) Visuokinetic activities of primate prefrontal neurons during delayed-response performance. $\mathrm{J}$ Ncurophysiol 37:1197-1212.

Mackworth JF (1970) Vigilance and attention. Baltimore: Penguin.

Mackworth NH (1948) The breakdown of vigilance during prolonged visual search. Q J Exp Psychol 1:6-21.

Mackworth NH (1957) Some factors affecting vigilance. Adv Sci 53: 389-393.

Miyashita Y, Rolls ET, Cahusac PM, Niki H, Feigenbaum JD (1989) Activity of hippocampal neurons in the monkey related to a conditional spatial response task. J Neurophysiol 61:669-678.

Miyawaki T, Kawamura H, Komatsu K, Yasugi T (1991) Chemical stimulation of the locus coeruleus: inhibitory effects on hemodynamics and renal sympathetic nerve activity. Brain Res 568:101-108.

Miyawaki T, Kawamura H, Hara K, Suzuki K, Usui W, Yasugi T (1993) Differential regional hemodynamic changes produced by L-glutamate stimulation of the locus coeruleus. Brain Res 600:56-62.

Mohammed AK, Callenholm NE, Järbe TU, Swedberg MD, Danysz W, Robbins TW, Archer T (1986) Role of central noradrenaline neurons in the contextual control of latent inhibition in taste aversion learning. Behav Brain Res 21:109-118.

Moore RY, Bloom FE (1979) Central catecholamine neuron systems: anatomy and physiology of the norepinephrine and epinephrine systems. Annu Rev Neurosci 2:113-168.

Nelson RJ, McCandlish CA, Douglas VD (1990) Reaction times for hand movements made in response to visual versus vibratory cues. Somatosens Mot Res 7:337-352.

Parasuraman R (1984) The psychobiology of sustained attention. In: Sustained attention in human performance (Warm JS, ed), pp 61101. New York: Wiley.

Parasuraman R, Davies DR (1976) Decision theory analysis of response latencies in vigilance. J Exp Psychol 2:578-590.

Petersen SE, Robsinson DL, Keys W (1985) Pulvinar nuclei of the behaving rhesus monkey: visual responses and their modulation. $J$ Neurophysiol 54:867-886.

Pieribone VA, Aston-Jones G (1988) The iontophoretic application of fluoro-gold for the study of afferents to deep brain nuclei. Brain Res 475:259-271.

Pieribone VA, Aston-Jones G (1991) Adrenergic innervation of the rat nucleus locus coeruleus arises from the $\mathrm{Cl}$ and $\mathrm{C} 3$ cell groups in the rostral medulla: an anatomic study combining retrograde transport and immunofluorescence. Neuroscience 41:525-542.

Pineda JA, Foote SL, Neville HJ (1989) Effects of locus coeruleus lesions on auditory, long-latency, event-related potentials in monkey J Neurosci 9:81-93.

Posner M, Petersen S (1990) The attention system of the human brain. Annu Rev Ncurosei 13:25-42.

Posner ME (1980) Orienting of attention. Q J Exp Psychol 32:3-25.

Posner MI (1992) Attention as a cognitive and neural system. Curr Direct Psychol Sci 1:11-14.

Posner MI (1993) Interaction of arousal and selection in the posterior attention network. In: Attention: selection, awareness and control. A tribute to Donald Broadbent (Baddeley A, Weiskrantz L, eds), pp 390-405. New York: Oxford UP. 
Pritchard WS (1981) Psychophysiology of P300. Psychol Bull 89:506540.

Rajkowski J, Kubiak P, Aston-Jones G (1992) Activity of locus coeruleus neurons in behaving monkeys varies with focussed attention: short- and long-term changes. Soc Neurosci Abstr 18:538.

Rajkowski J, Kubiak P, Aston-Jones G (1993) Correlations between locus coeruleus (LC) neural activity, pupil diameter and behavior in monkey support a role of LC in attention. Soc Neurosci Abstr 19: 974.

Rasmussen K, Jacobs BL (1986) Single unit activity of locus coeruleus neurons in the freely moving cat. II. Conditioning and pharmacologic studies. Brain Res 371:335-344.

Rasmussen K, Morilak DA, Jacobs BL (1986) Single unit activity of locus coeruleus neurons in the freely moving cat. I. During naturalistic behaviors and in response to simple and complex stimuli. Brain Res 371:324-334.

Redmond JDE (1979) New and old evidence for the involvement of a brain norepinephrine system in anxiety. In: Phenomenology and treatment of anxiety (Fann WE, ed), pp 153-203. New York: Spectrum.

Reiner PB (1986) Correlational analysis of central noradrenergic neuronal activity and sympathetic tone in behaving cats. Brain Res 378: $86-96$.

Robinson DL, Baizer JS, Dow BM (1980) Behavioral enhancement of visual responses of prestriate neurons of the rhesus monkey. Invest Ophthalmol Vis Sci 19:1120-1123.
Sara SJ, Segal M (1991) Plasticity of sensory responses of locus coeruleus neurons in the behaving rat: implications for cognition. Prog Brain Res 88:571-585

Siever LJ, Davis KL (1985) Overview: toward a dysregulation hypothesis of depression. Am J Psychiatry 142:1017-1031.

Svensson TH, Bunney RS, Aghajanian GK (1975) Inhibition of both noradrenergic and serotonergic neurons in brain by the alpha-adrenergic antagonist, clonidine. Brain Res 92:291-306.

Thorpe SJ, Rolls ET, Maddison S (1983) The orbitofrontal cortex: neuronal activity in the behaving monkey. Exp Brain Res 49:93-115.

Ungerstedt $U$ (1971) Stereotaxic mapping of the monoamine pathways in the rat brain. Acta Physiol Scand [Suppl] 367:1-48.

Valentino RJ, Curtis AL (1991) Antidepressant interactions with corticotropin-releasing factor in the noradrenergic nucleus locus coeruleus. Psychopharmacol Bull 27:263-269.

Ward DG, Gunn CG (1976) Locus coeruleus complex: elicitation of a pressor response and a brain stem region necessary for its occurrence. Brain Res 107:401-406.

Warm JS, Jerison HJ (1984) The psychophysics of vigilance. In: Sustained attention in human performance (Warm JS, ed), pp 15-59. New York: Wiley.

Wurtz RH, Mohler CW (1976) Enhancement of visual responses in monkey striate cortex and frontal eye fields. J Neurophysiol 39:766772. 